\title{
Quantum Radiation Reaction: From Interference to Incoherence
}

\author{
Victor Dinu, ${ }^{1, *}$ Chris Harvey, ${ }^{2, \dagger}$ Anton Ilderton, ${ }^{2, \ddagger}$ Mattias Marklund, ${ }^{2, \S}$ and Greger Torgrimsson ${ }^{2, \|}$ \\ ${ }^{1}$ Department of Physics, University of Bucharest, P.O. Box MG-11, Măgurele 077125, Romania \\ ${ }^{2}$ Department of Physics, Chalmers University of Technology, SE-41296 Gothenburg, Sweden
}

(Received 25 September 2015; published 29 January 2016)

\begin{abstract}
We investigate quantum radiation reaction in laser-electron interactions across different energy and intensity regimes. Using a fully quantum approach which also accounts exactly for the effect of the strong laser pulse on the electron motion, we identify in particular a regime in which radiation reaction is dominated by quantum interference. We find signatures of quantum radiation reaction in the electron spectra which have no classical analogue and which cannot be captured by the incoherent approximations typically used in the high-intensity regime. These signatures are measurable with presently available laser and accelerator technology.
\end{abstract}

DOI: 10.1103/PhysRevLett.116.044801

Intense light sources offer new prospects for observing quantum effects in laser-matter interactions. Phenomena such as particle beam spreading [1], cooling [2,3] and trapping $[4,5]$ can all be phrased in terms of the quantum recoil experienced by particles interacting with laser pulses, recoil which dominates particle motion in certain regimes [6]. Because of this the topic of quantum recoil, also called quantum radiation reaction ("QRR"), now receives a great deal of attention [7-12].

Investigations of QRR often focus on high-intensity regimes currently out of experimental reach. In such regimes QRR comes from multiphoton emission, and the shortness of the "formation length" of quantum processes at high intensity implies that these emissions can be described as incoherent events $[13,14]$. In this Letter we show that the nature of QRR varies significantly in different intensity and energy regimes, in particular regimes which are relevant to experiments soon to be performed. In particular we reveal a regime, accessible with the laser intensities and accelerator technology available today, in which QRR is dominated by coherent quantum effects with no classical analogue, effects which are distinct from those in the high-intensity regime and which cannot be described by the approximations or numerical methods used there. Further, we will find new kinematic delineations of the different regimes.

Consider an electron interacting with a strong electromagnetic field. The classical Lorentz force equation predicts that the electron moves with some momentum $\pi_{\mu}$. A measurement of the electron momentum would, however, yield a different result $P_{\mu}$, because the Lorentz equation does not account for the fact that the electron radiates and, by conservation of momentum, recoils when it does so [15]. The impact of this radiation reaction ("RR") on the motion of the electron can be characterised simply by the difference between the actual momentum of the electron and that predicted by the Lorentz force: $P_{\mu}-\pi_{\mu}$ is classical RR. The momentum $P_{\mu}$ can be obtained as the classical or low-energy limit of a quantum mechanical observable, namely the expectation value of the electron momentum operator $\hat{P}_{\mu}[16-19]$. Hence $\left\langle\hat{P}_{\mu}\right\rangle-\pi_{\mu}$ is a measure of QRR. The expectation value $\left\langle\hat{P}_{\mu}\right\rangle$ can be calculated for arbitrary weak fields in perturbation theory [16] but this is not sufficient for our purposes as the fields of interest are strong. In order to account fully for the impact of a strong laser field on electron motion, as well as giving a fully quantum treatment of $\left\langle\hat{P}_{\mu}\right\rangle$ in QED, we begin with a plane wave laser model. This is satisfactory in the high-energy regime we consider first, while beam focusing at high intensity will be accounted for below. The QED calculation of $\left\langle\hat{P}_{\mu}\right\rangle$ follows [19] and is described in the Supplemental Material [20].

QRR effects depend on the following parameters. Let $\omega$ and $k_{\mu}$ be typical laser frequency and momentum scales, and let $p_{\mu}$ be the initial electron momentum. Then the energy scale of the interaction is $b_{0} \equiv k \cdot p / m^{2}$ which is $\simeq 2 \omega \gamma / m$ for large $\gamma$. (We use units such that $\hbar=c=1$ throughout.) Quantum effects in a field $F_{\mu \nu}$ are often characterized using the "quantum efficiency parameter" $\chi=\sqrt{p \cdot(e F)^{2} \cdot p} / m^{3}$. For a wave of intensity $a_{0}=e E / m \omega$, field strength $E, \chi$ becomes the product $\chi=$ $a_{0} b_{0}$ [13]. Hence a given $\chi$ may be achieved through different combinations of intensity and energy, and we will see that different choices lead to very different physics. We take the laser to propagate in the $z$ direction and be polarized in the $x$ direction, so that the laser fields depend on the phase $\phi \equiv \omega(t+z)$ through a potential with $x$-component $e A^{x}=m a_{0} e^{-\phi^{2} / \tau^{2}} \sin (\phi)$. We fix the wavelength at $\lambda=2 \pi / \omega=820 \mathrm{~nm}$ and choose $\tau$ such that the FWHM pulse duration is $15 \mathrm{fs}$.

We begin our investigation with achievable parameters $a_{0}=1$, corresponding to an intensity of $\sim 10^{18} \mathrm{~W} / \mathrm{cm}^{2}$, and $\gamma=10^{5}$ [31], suggesting a maximum $\chi=b_{0}=0.59$. In Fig. 1 we plot, for a head-on collision, the electron 


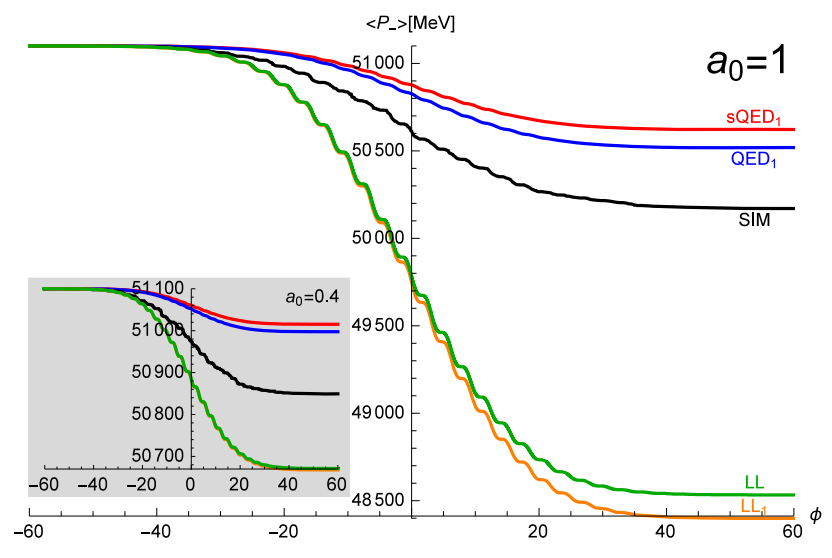

FIG. 1. QRR in the interference-dominated regime, $a_{0}=1$ and $\gamma=10^{5}$. Red/blue: scalar QED/QED exact to order $\alpha$ (subscript 1). Black/"SIM": simulation data based on the LCA. Green: exact solution of the classical LL equation. Orange/" $\mathrm{LL}_{1}$ ": the classical limit of the first order QED results. Inset: curves for $\gamma=10^{5}, a_{0}=0.4$, and $\chi=0.24$.

momentum component $P_{-} \equiv\left(E-p_{z}\right) / 2$, the difference between energy and $z$ momentum, which shows the most significant deviation from the Lorentz-force result; $P_{-}$is conserved without RR, but recoil effects break this symmetry [32,33]. It is convenient to consider $\left\langle\hat{P}_{\mu}\right\rangle$ as a function of phase $\phi$, as this relates the momentum to the local intensity in the laser pulse. (Collision at $45^{\circ}$ incidence, as may be experimentally necessary, can be advantageous as it makes QRR visible in all momentum components; for examples see the Supplemental Material [20].)

Note first that Fig. 1 shows only a small (5\%) difference between the exact solution of the classical Landau-Lifshitz ("LL") equation [32] and the classical limit of the QED result (giving the first order solution of the LL and LAD equations [16-19]). This suggests that higher-order multiphoton effects are small. However, classical predictions are invalid here: accounting for quantum effects clearly shows that the classical theory greatly overestimates RR losses, the relative error being around 350\%. The inset in Fig. 1 shows that quantum effects persist even for smaller $\chi$ [34]. Our QED approach allows us to account fully for spin, and the figure shows that spin slightly increases radiative losses relative to those in scalar QED [35]. Figure 1 also shows results from by-now standard numerical simulations of intense laser-matter interactions which assume Lorentz force propagation between quantum emissions described in a locally constant approximation ("LCA") [36-39]. The approximations behind the codes hold only for $a_{0} \gg 1$, so they should not be expected to recover QRR in the considered regime; indeed the simulation data in Fig. 1, obtained from $10^{4}$ runs, fail to fully capture quantum effects.

To understand these results, in particular the quantum reduction of energy loss due to $\mathrm{RR}$, we examine the structure of the average momentum $\left\langle\hat{P}_{\mu}\right\rangle$. To first order in $\alpha$ (the fine structure constant) and exactly in all other parameters, $\left\langle\hat{P}_{\mu}\right\rangle$ may be written, for an arbitrary pulse shape and duration, as

$$
\left\langle\hat{P}_{\mu}\right\rangle(\phi)=\pi_{\mu}(\phi)+\int_{-\infty}^{\phi} d \varphi \int_{0}^{\infty} \mathrm{d} \theta \mathcal{F}_{\mu}(\phi, \varphi, \theta),
$$

in which $\mathcal{F}_{\mu}$ is given explicitly in the Supplemental Material [20]; the details are not needed here. The important argument is $\theta$, which is the difference between phases at which photon emission occurs in the quantum state of the radiating system, and its complex conjugate. The $\theta$ integral contains quantum interference effects and is purely quantum mechanical, as it is confirmed by considering the low energy limit $b_{0} \ll 1$. In this limit the integrand collapses to a delta function in $\theta$ [19], exhibiting decoherence [40] and leading to a purely local expression in agreement with classical predictions [16-19,41]. Importantly, the classical limit of (1) is closely related to the high-intensity limit. For high intensity (made precise below) the $\theta$ integrand is dominated by small perturbations around the classical point $\theta \simeq 0$. These semiclassical contributions give the LCA to $\langle\hat{P}\rangle$ at high intensity. By analyzing the momentum for arbitrary pulse shapes we show in the Supplemental Material [20] that the high-intensity and classical regimes are collectively characterized by the restriction

$$
\frac{1+a_{0}^{2}}{b_{0}} \gg 1 \text {. }
$$

This gives a kinematic refinement of the usual statement that only $a_{0} \gg 1$ is required for the LCA to hold [42]. (The regime $a_{0}>1$ and $a_{0}^{2}>b_{0}$ has also been identified as that of the "quantum synchrotron approximation" [43].) For other refinements coming from consideration of the emitted photon spectrum see [44]. For ultra-intense optical lasers and achievable electron energies, (2) clearly implies $a_{0} \gg 1$, but if either the energy is high or if the intensity is not so high so that (2) is not satisfied, quantum RR must be described using the full coherent expression (1). This integral contains correlations and interference between scattering events separated by arbitrarily large phase differences; it is this quantum interference which reduces RR energy losses as compared to the classical theory. Hence both the classical theory, which misses all interference effects, and the LCA, which captures only "short range" interference effects but misses the long range effects, overestimate RR losses.

We can now explain the behaviors seen in Fig. 1. The inequality (2) is not fulfilled: because $b_{0}$ is not small enough and $a_{0}$ is not large enough, neither a low energy (local) nor a high-intensity (locally constant) approximation is valid. Rather the quantum interference effects in the coherent double integral in (1) are needed to properly capture QRR; when this holds we say that we are in an "interference dominated regime" (IDR). The simulation results in Fig. 1 naturally overestimate the energy loss as they are based on the LCA, which misses quantum 


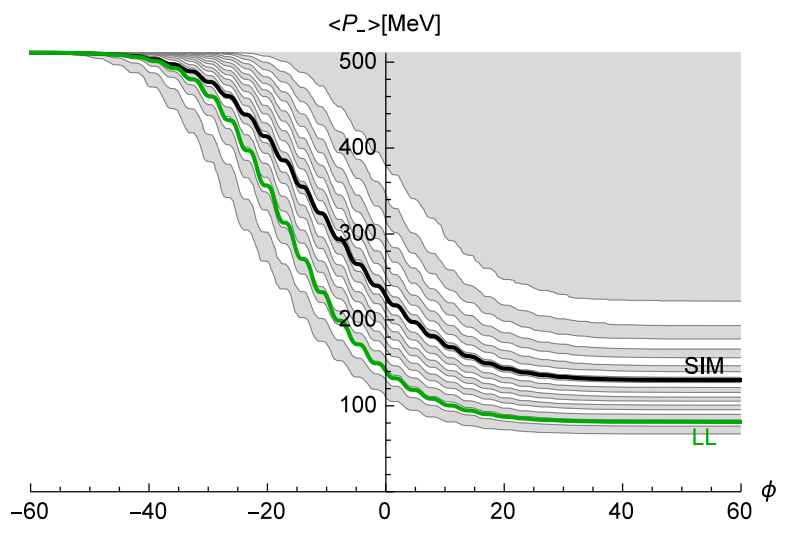

FIG. 2. $P_{-}$for $a_{0}=100, \gamma=10^{3}$ (high-intensity regime). The relative error in the classical energy loss (green) compared to the quantum multiphoton description (black) is only $15 \%$. Grey/white bands illustrate the momentum distribution calculated with the numerical approach: each band contains, from top to bottom, $5 \%, 10 \%, 15 \%$... of all trajectories.

interference. This is consistent with recent investigations which show that the LCA misses spectral features which depend on long distance phase correlations or interference from multiple stationary points, in photon emission [45,46], pair production [47-49], and ionization [50].

We will now compare and contrast the IDR with the high-intensity regime accessible by the ELI-NP facility [51]. Taking $a_{0}=100$ and $\gamma=10^{3}$ gives the same $\chi$ as above, but in a different regime where (2) is satisfied. The LCA should therefore provide a good approximation here, in a regime where interference effects are suppressed and QRR comes from multiple incoherent photon emission [14], and the numerical approach is on firm ground. [Entering an IDR for $a_{0} \gg 1$ would, from (2), require extremely high energy particles.] Results are shown in Fig. 2. An average of 28.45 photons were emitted over $10^{4}$ simulation runs; higher-order multiphoton effects are indeed important. For this reason the order- $\alpha$ QED result is insufficient to capture the correct physics, and therefore not shown. Figure 2 shows that the difference between quantum and classical results is not large; the relative error in the classical prediction (an overestimate) is around 15\%, compared with around $350 \%$ in the IDR. The reason for this is the high intensity; the system is driven back toward the classical regime as particles are shaken violently by the laser and very quickly radiate away their initial energy, well before reaching the peak field. The maximum $\chi$ achieved is (from simulation data) $\chi \simeq 0.25$, despite the initial parameters giving us a theoretical maximum $\chi \simeq 0.59$. This resistance to entering the high-intensity, high-energy regime is well known [52,53] and is responsible for e.g. hindering comparisons of different classical RR models [54]. In all our high $a_{0}$ simulations the number $N$ of photons emitted per laser cycle is consistent with the estimate, derived assuming a formation length $\sim 1 /\left(\omega a_{0}\right)$

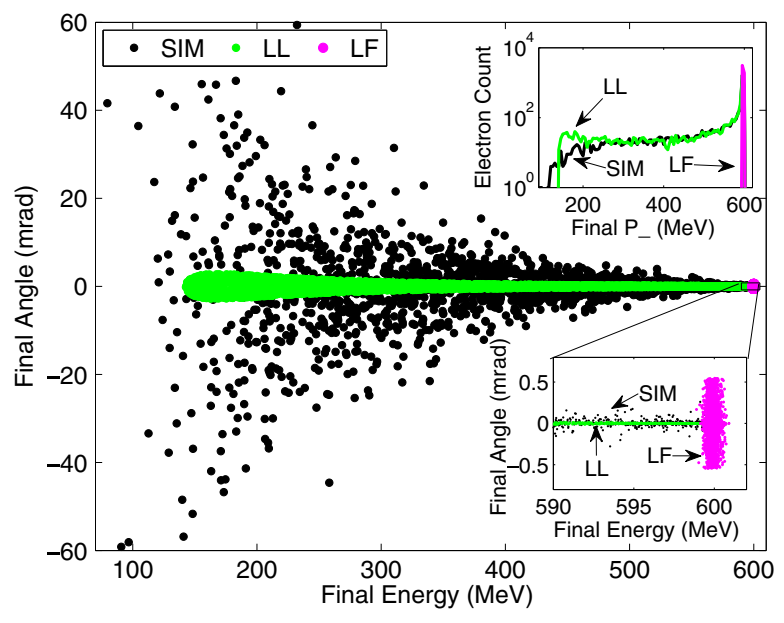

FIG. 3. Scattered electron spectrum for ELI-NP parameters, as in the text. We look along the energy axis to distinguish between classical models with and without RR, and along the angular scattering axis to distinguish between classical and quantum RR.

[13], $N \sim \sqrt{2} \pi \alpha a_{0}\left(2 \pi \alpha a_{0}\right)$ for linear (circular) polarization which differs from the commonly used $N \sim \alpha a_{0}$.

We turn now to two specific experimental scenarios in which signals of QRR will be sought in different regimes. The first extends the calculation above to a fully realistic collision of an electron beam with a focused laser pulse, taking account of longitudinal and transverse beam structures and using the planned parameters of ELI-NP. We simulated a bunch of 5000 electrons with average energy $600 \mathrm{MeV}(\gamma \simeq 1200) \pm 0.1 \%$ and transverse/longitudinal spread of FWHM $15 \mu \mathrm{m} / 400 \mathrm{pm}$ colliding with a focused Gaussian pulse of wavelength $\lambda=820 \mathrm{~nm}$, focal spot radius $w_{0}=5 \mu \mathrm{m}$, FWHM pulse length $22 \mathrm{fs}$ and peak intensity $10^{22} \mathrm{~W} / \mathrm{cm}^{2}\left(a_{0} \simeq 70\right)$. The beam profiles are shown in the Supplemental Material [20]. Three simulations were performed, in which recoil effects were either neglected entirely (motion described only by the Lorentz force), treated classically (motion described by the Landau Lifshitz equation) or treated quantum mechanically using the numerical approach [36-39]. The results in Fig. 3 show marked differences between the three models.

Looking along the energy axis shows that both classical and quantum RR cause the electron beam to emerge from the pulse with an energy spread of several hundreds of $\mathrm{MeV}$, whereas neglecting recoil effects implies that the electrons essentially retain their initial energies [2,3]. However distinguishing quantum and classical contributions to this effect is difficult; see also the top inset of Fig. 3. This is because beam focusing (finite width with varying intensity) gives an impact-parameter spread in energy which acts as a background. Looking instead along the vertical axis, corresponding to transverse scattering angle, we see that the quantum electrons develop a transverse spread spanning several degrees, corresponding to a transverse momentum spread of around $10 \mathrm{MeV}$, whereas the 


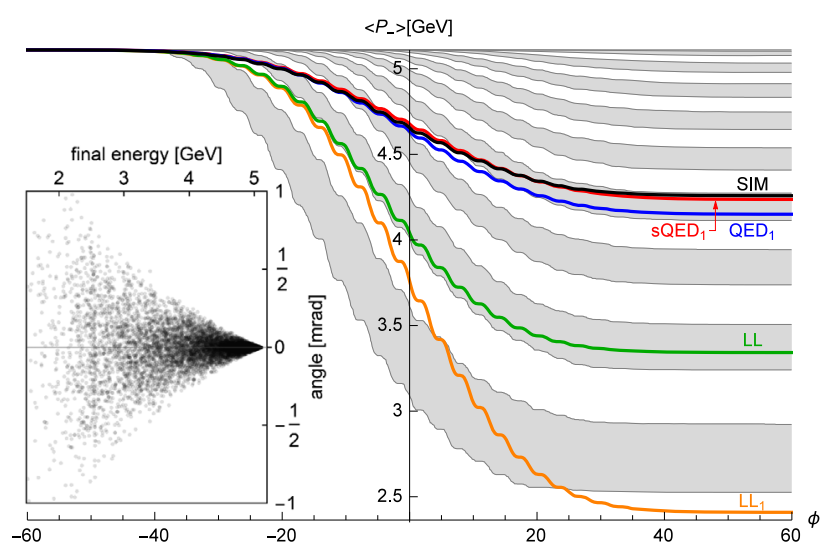

FIG. 4. $\left\langle P_{-}\right\rangle$for a head-on collision in the crossover regime, colors as above. $a_{0}=10$ and $\gamma=10^{4}$. Just over $60 \%$ of trajectories show lower-than-average energy loss. The inset shows the final distribution of electron energies vs scattering angle, due to stochastic quantum effects.

classically modeled electrons remain largely confined to the beam axis. The stochastic nature of quantum emissions means that the electron bunch will diffuse in transverse momentum space [1], whereas the classical model only allows a net loss of transverse momentum in the radiating electrons, with the exception of comparatively much smaller ponderomotive effects (a small background) arising from beam focusing. Hence the transverse spreading provides a measurable signature of quantum RR distinct from its classical counterpart. (Transverse size effects are expected to be subleading in the IDR with high energy particles: the highest energy emissions come from particles on beam axis [55] and transverse deflection from the plane wave trajectory is suppressed by factors of $a_{0} / \gamma \ll 1$ [56].)

Finally, consider the regime $a_{0} \sim 1-10$ and $\gamma \leq 10^{4}$ which should be accessible on the Bella [57] and Gemini [58,59] lasers. Toward the lower (upper) extreme of the energy range classical (quantum) effects are significant. Toward the lower (upper) extreme of the intensity range the LCA fails (works), so that simulations are less (more) reliable. At the same time the lowest order QED results become more (less) reliable, because the longer or more intense the pulse, the more higher-order corrections are required to account for multiple photon emissions in order to give the correct rate of energy loss. In this "crossover" regime it is therefore necessary to account carefully for the possibility of both multiphoton and interference effects. This regime is distinct from the IDR and high-intensity regimes above. It is theoretically challenging, as higher-order corrections are difficult to calculate analytically when the constraint (2) is not fulfilled [19].

An example of the electron momentum in the crossover regime is shown in Fig. 4 for parameters giving the same $\chi$ as above. Here the LCA is sufficient to capture the physics - the LCA to (1) is indistinguishable from the full result on the scale shown. Since an average of 2.77

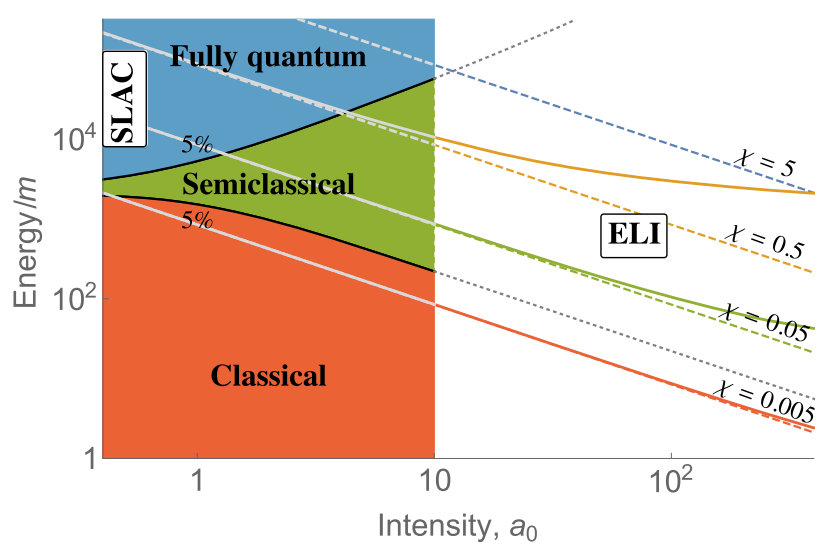

FIG. 5. Characterizing radiation reaction in the energy-intensity plane. Two types of effects are shown, to the left and right of the division at $a_{0}=10$ which separates, very approximately, the applicability of the methods used. The classical (semiclassical/ locally constant) approximation to RR differs by less than 5\% from the full, coherent, quantum integral (1) in the regions marked "classical" ("semiclassical") on the left of the plot. As intensity increases, higher energies are needed to access fully quantum effects. To the right, dotted lines are those of constant "ideal" $\chi$ calculated from peak intensity and initial energy, while solid lines are those of constant peak $\chi$ taking into account classical cooling effects modeled by the LL equation. The existence of the different regimes and cooling effects are general, though their precise form depends on pulse shape. Labels in white boxes indicate the approximate operating regimes of the named facilities [31,51].

photons were emitted over $10^{4}$ runs we should expect a discrepancy between the simulation and order- $\alpha$ QED results due to multiphoton effects. Despite this, we find that they are in close mutual agreement (and both differ significantly from the classical prediction). Remarkably, the same agreement is found for all other parameters we have examined in this regime. The fact that the two very different approaches agree across an energy and intensity range relevant to upcoming experiments, e.g. on Gemini, is extremely encouraging. We stress though that further investigation of this interesting regime is needed to ensure that the correct result is obtained.

One reason for the smallness of the expected discrepancy in Fig. 4 can be seen by from the grey bands: most electron trajectories stay distributed close to the Lorentz-force trajectory for most of the pulse. The distribution and spread of momenta is therefore an interesting topic for further study, along with the quantum mechanical variance $\left\langle\hat{P}^{2}\right\rangle-\langle\hat{P}\rangle^{2}$. The inset in Fig. 4 shows the stochastic spreading of on-axis electrons due to purely quantum effects, cf. Fig. 3. This is one of the experimental signatures of QRR which will be investigated at high-power laser facilities over the coming years.

To conclude, we have examined QRR effects in different energy and intensity regimes. Figure 5 illustrates these regimes and our results. We have seen that interference effects, completely absent in classical physics, reduce 
energy losses relative to classical predictions and contribute significantly to QRR for high energy and not too high intensity. This "interference dominated regime," or IDR, stands in contrast to the high-intensity regime in which quantum RR is essentially semiclassical and captured by a locally constant approximation, and where large cooling effects draw systems back toward the classical regime [60]. We have also identified a kinematic delineation of these different regimes, see also [43,61]; refinements accounting for pulse duration [62] or final state kinematics [44] are interesting topics for future study.

Concerning experimental signatures, we have confirmed that both classical and quantum radiation reaction will be visible in high-intensity ELI-NP experiments. We have also highlighted a "crossover regime" where both multiphoton and quantum interference effects are significant. This is the most interesting, theoretically challenging, and perhaps experimentally urgent regime.

The authors are supported by a Strategic Grant POSDRU/159/1.5/S/137750 (V.D.), the Olle Engkvist Foundation, Grant No. 2014/744 (A. I.), the Wallenberg Foundation project "Plasma based compact ion sources" (A. I., M. M.), and the Swedish Research Council, Grants No. 2011-4221 (A. I., G. T), No. 2012-5644, and No. 20134248 (C. H., M. M).

*dinu@barutu.fizica.unibuc.ro

†christopher.harvey@chalmers.se

*anton.ilderton@chalmers.se

\$mattias.marklund@chalmers.se

"greger.torgrimsson@ chalmers.se

[1] D. G. Green and C. N. Harvey, Phys. Rev. Lett. 112, 164801 (2014).

[2] N. Neitz and A. Di Piazza, Phys. Rev. Lett. 111, 054802 (2013).

[3] S. R Yoffe, Y. Kravets, A. Noble, and D. Jaroszynski, New J. Phys. 17, 053025 (2015).

[4] A. Gonoskov et al., Phys. Rev. Lett. 113, 014801 (2014).

[5] L. L. Ji, A. Pukhov, I. Yu. Kostyukov, B. F. Shen, and K. Akli, Phys. Rev. Lett. 112, 145003 (2014).

[6] S. V. Bulanov, T. Esirkepov, J. Koga, and T. Tajima, Plasma Phys. Rep. 30, 196 (2004); Phys. Plasmas 12, 073103 (2005).

[7] A. Di Piazza, C. Muller, K. Z. Hatsagortsyan, and C. H. Keitel, Rev. Mod. Phys. 84, 1177 (2012).

[8] J-X. Li, K.Z. Hatsagortsyan, and C. H. Keitel, Phys. Rev. Lett. 113, 044801 (2014).

[9] M. Vranic, J. L. Martins, J. Vieira, R. A. Fonseca, and L. O. Silva, Phys. Rev. Lett. 113, 134801 (2014).

[10] T. G. Blackburn, C. P. Ridgers, J. G. Kirk, and A. R. Bell, Phys. Rev. Lett. 112, 015001 (2014).

[11] H. Y. Wang, X. Q. Yanm, and M. Zepf, Phys. Plasmas 22, 093103 (2015).

[12] M. Vranic, T. Grismayer, R. A. Fonseca, and L. O. Silva, arXiv:1511.04406.

[13] V. I. Ritus, J. Russ. Laser Res. 6, 497 (1985).
[14] A. Di Piazza, K. Z. Hatsagortsyan, and C. H. Keitel, Phys. Rev. Lett. 105, 220403 (2010).

[15] H. A. Lorentz, The Theory of Electrons (Teubner, Leipzig, 1909); , Theorie der Elektrizität (Teubner, Leipzig, 1905); P. A. M. Dirac, Proc. R. Soc. A 167, 148 (1938).

[16] V. S. Krivitsky and V. N. Tsytovich, Usp. Fiz. Nauk 161, 125 (1991) [Sov. Phys. Usp. 34, 250 (1991)].

[17] A. Higuchi, Phys. Rev. D 66, 105004 (2002); 69, 129903(E) (2004).

[18] A. Higuchi and G. D. R. Martin, Phys. Rev. D 70, 081701 (2004); Phys. Rev. D 73, 025019 (2006).

[19] A. Ilderton and G. Torgrimsson, Phys. Lett. B 725, 481 (2013); Phys. Rev. D 88, 025021 (2013).

[20] See Supplemental Material at http://link.aps.org/ supplemental/10.1103/PhysRevLett.116.044801, which includes Refs. [21-30], for the derivation of Eq. (1), plots of $45^{\circ}$ collisions, and more information on Fig. 3.

[21] R. A. Neville and F. Rohrlich, Phys. Rev. D 3, 1692 (1971).

[22] B. R. Holstein and J. F. Donoghue, Phys. Rev. Lett. 93, 201602 (2004).

[23] S. J. Brodsky and P. Hoyer, Phys. Rev. D 83, 045026 (2011).

[24] T. Heinzl et al., Phys. Rev. E 91, 023207 (2015).

[25] F. Hebenstreit, A. Ilderton, M. Marklund, and J. Zamanian, Phys. Rev. D 83, 065007 (2011).

[26] V. N. Baier and V. M. Katkov, Sov. Phys. JETP 26, 854 (1968).

[27] A. I. Nikishov and V. I. Ritus, Zh. Eksp. Teor. Fiz. 46, 776 (1963) [Sov. Phys. JETP 19, 529 (1964)].

[28] M. Boca and V. Florescu, Phys. Rev. A 80, 053403 (2009).

[29] V. Dinu, T. Heinzl, and A. Ilderton, Phys. Rev. D 86, 085037 (2012).

[30] D. Seipt, S. G. Rykovanov, A. Surzhykov, and S. Fritzsche, Phys. Rev. A 91, 033402 (2015).

[31] C. Bamber et al., Phys. Rev. D 60, 092004 (1999).

[32] A. Di Piazza, Lett. Math. Phys. 83, 305 (2008).

[33] C. Harvey, T. Heinzl, and M. Marklund, Phys. Rev. D 84, 116005 (2011).

[34] Low-intensities are also relevant in the context of cascade initiation by high-energy particles entering the low- $a_{0}$ part of laser pulses; see A. A. Mironov, N. B. Narozhny, and A. M. Fedotov, Phys. Lett. A 378, 3254 (2014).

[35] It may be interesting to compare with the spin dependence of pair production in fields with large interference effects; see C. K. Dumlu and G. V. Dunne, Phys. Rev. D 83, 065028 (2011).

[36] N. V. Elkina, A. M. Fedotov, I. Y. Kostyukov, M. V. Legkov, N. B. Narozhny, E. N. Nerush, and H. Ruhl, Phys. Rev. ST Accel. Beams 14, 054401 (2011).

[37] C. P. Ridgers, J. G. Kirk, R. Duclous, T. G. Blackburn, C. S. Brady, K. Bennett, T. D. Arber, and A. R. Bell, J. Comput. Phys. 260, 273 (2014).

[38] D. G. Green and C. N. Harvey, Comput. Phys. Commun. 192, 313 (2015).

[39] A. Gonoskov et al., Phys. Rev. E 92, 023305 (2015).

[40] W. H. Zurek, Rev. Mod. Phys. 75, 715 (2003).

[41] E. J. Moniz and D. H. Sharp, Phys. Rev. D 15, 2850 (1977).

[42] The origin of the numerator in the constraint (2) is the generalized intensity-dependent mass; see T. W. B. Kibble, A. Salam, and J. A. Strathdee, Nucl. Phys. B96, 255 (1975); 
C. Harvey, T. Heinzl, A. Ilderton, and M. Marklund, Phys. Rev. Lett. 109, 100402 (2012).

[43] A. K. Khokonov and M. K. Khokonov, Tech. Phys. Lett. 31, 154 (2005).

[44] A. K. Khokonov, M. K. Khokonov, and A. A. Kizdermishov, Tech. Phys. 47, 1413 (2002).

[45] C. N. Harvey, A. Ilderton, and B. King, Phys. Rev. A 91, 013822 (2015).

[46] D. Seipt, A. Surzhykov, S. Fritzsche, and B. Kämpfer, arXiv: 1507.08868 .

[47] S. Meuren, C. H. Keitel, and A. Di Piazza, arXiv:1503.03271.

[48] T. Nousch, D. Seipt, B. Kämpfer, and A. I. Titov, arXiv:1509.01983.

[49] M. J. A. Jansen and C. Müller, arXiv:1511.07660.

[50] A. S. Maxwell and C. Figueira de Morisson Faria, Phys. Rev. A 92, 023421 (2015).

[51] Extreme Light Infrastructure-Nuclear Physics facility, Bucharest-Magurele, Romania. http://www.eli-np.ro/.

[52] I. Ya. Pomeranchuk, Zh. Eksp. Teor. Fiz. 9, 915 (1939).
[53] A. M. Fedotov, N. V. Elkina, E. G. Gelfer, N. B. Narozhny, and H. Ruhl, Phys. Rev. A 90, 053847 (2014).

[54] Y. Kravets, A. Noble, and D. Jaroszynski, Phys. Rev. E 88, 011201(R) (2013).

[55] F. Mackenroth, A. Di Piazza, and C. H. Keitel, Phys. Rev. Lett. 105, 063903 (2010).

[56] A. Di Piazza, Phys. Rev. Lett. 113, 040402 (2014).

[57] W. P. Leemans, R. Duarte, E. Esarey, S. Fournier, C. G. R. Geddes, D. Lockhart, C. B. Schroeder, C. Toth, J. L. Vay, and S. Zimmermann, AIP Conf. Proc. 1299, 3 (2010).

[58] Gemini at the CLF, http://www.clf.stfc.ac.uk.

[59] G. Sarri et al., Phys. Rev. Lett. 113, 224801 (2014).

[60] Our results are consistent with the result that the classical emission spectrum of an electron in a plane wave is an upper bound for the quantum spectrum; see C. Harvey, T. Heinzl, and A. Ilderton, Phys. Rev. A 79, 063407 (2009); V. Dinu, Phys. Rev. A 87, 052101 (2013).

[61] S. V. Bulanov et al., Proc. SPIE Int. Soc. Opt. Eng. 8780, 878015 (2013).

[62] B. King and H. Ruhl, Phys. Rev. D 88, 013005 (2013). 